\title{
A novel probabilistic distance measure for picture fuzzy sets with its application in classification problems
}

\author{
Abhishek Guleria (D), Rakesh Kumar Bajaj* (1) \\ Department of Mathematics, Jaypee University of Information Technology, Waknaghat, Solan, \\ Himachal Pradesh, India
}

\begin{abstract}
In the present communication, we propose the probabilistic distance measure for picture fuzzy sets where the probability of occurrence/non-occurrence of the picture fuzzy event have been incorporated. This framework has been clearly addressed through outline of a formulated problem and its probable solution structure along with its proof of validity. Further, the proposed probabilistic distance measure has been utilized to present an algorithm for solving some classification decision making problems in a more generalized way. Some important illustrative examples related to the problem of classification - building material classification, mineral classification and a decision making problem of financial investment risk have been worked out to exhibit the implementation of the proposed methodology. The obtained results have also been compared with the existing approaches of solving the classification problems. The uncertainty feature of the problem has been handled in a more broader sense reflecting the advantage of the introduced approach.
\end{abstract}

Mathematics Subject Classification (2010). 03E72, 15B15, 05C72, 05C76, 60A86

Keywords. picture fuzzy sets, distance measures, probabilistic randomness, uncertainty, classification problem

\section{Introduction}

The concept of distance measure plays a significant role in establishing a useful link from the expression of uncertain and imprecise information to various real world application fields such as decision making, clustering, financial risk analysis, and so on. In order to improve the basic and existing approaches of defining the distance measures, various researchers have duly paid attention by incorporating other determining factors. Initially, Zadeh $[41,42]$ introduced the concept of a fuzzy event and its probability as a natural extension of the classical probability of an event. Next, Atanassov [1] included the hesitancy part in a fuzzy event and extended the concept to intuitionistic fuzzy set so that the uncertainty inherent in many practical circumstances may be taken care with a broader range, particularly in the study of patterns and information systems. Essentially, such generalizations provide a formal way of dealing with real life problems in which the source

\footnotetext{
*Corresponding Author.

Email addresses: abhishekguleriahappy@gmail.com (A. Guleria), rakesh.bajaj@gmail.com (R.K. Bajaj)

Received: 21.01.2020; Accepted: 17.10.2020
} 
of impreciseness is the lack of sharply defined criteria of class membership instead of having the random variables. Szmidt and Kacprzyk [29] laid down the idea of connecting the concept of intuitionistic fuzzy event and its probability which is a consistent generalization of Zadeh's fuzzy event/probability measure.

Further, Yager [38] revealed that the existing structures of fuzzy set and intuitionistic fuzzy set is not capable enough to depict the human opinion in more practical/broader sense and introduced the notion of Pythagorean fuzzy set (PyFS) which effectively enlarged the span of information by introducing the new conditional constraint. PyFS is basically characterized by a membership value and a non-membership value such that the squared sum of these values is less than or equal to 1 . However, PyFS is more generalized than intuitionistic fuzzy set but there is another notion of uncertainty term called 'degree of refusal' which has not given due attention in a decision making process. This can be understood with the help of an example of 'voting system', where voters can be categorized into four different classes - one who votes for (yes), one who votes against (no), one who neither vote for nor against (abstain), one who refused for voting (refusal). It may be noted that the concept of 'refusal' is not being taken into account by any of the sets stated above. In order to deal with such circumstances and to develop a concept which would be sufficiently close to human's nature of flexibility, Cuong [5] introduced the concept of picture fuzzy set (PFS) in which all the four parameters, i.e., degree of membership, degree of indeterminacy (neutral), degree of nonmembership and the degree of refusal have been taken into account. Therefore, the generalization of fuzzy sets to picture fuzzy sets may be well understood by the geometric presentation in Figure 1 showing the better coverage of the imprecise information.

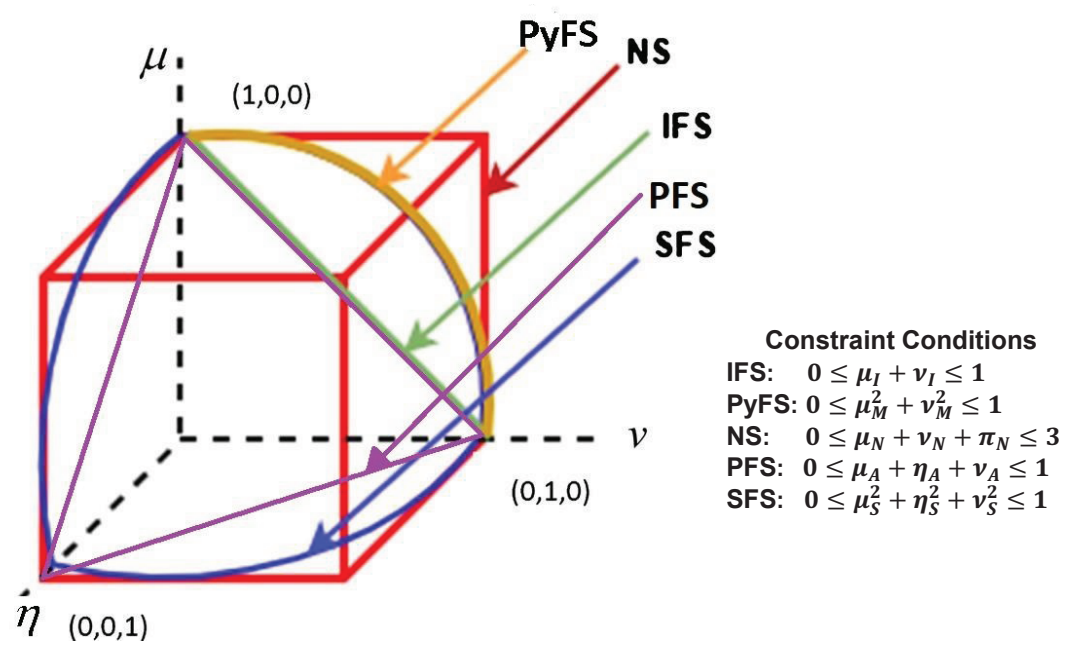

Figure 1. Extension of Fuzzy Set to Picture Fuzzy Set

Burillo and Bustince [4] studied the distance measures between intuitionistic fuzzy sets which express the information in terms of two parameters i.e., membership and nonmembership function. Further, Szmidt and Kacprzyk [30] studied some limitations of not taking into account the third parameter of uncertainty (hesitancy function) and developed some new distance measures between intuitionistic fuzzy sets. Based on the Hausdorff distance and $L_{p}$ metric, Hung and Yang $[18,19]$ studied some similarity measures/distance measures of intuitionistic fuzzy sets. Next, based on matrix norm and fuzzy implication, Hatzimichailidis et al. [17] proposed a new method for calculating the distance between intuitionistic fuzzy sets. Papakostas et al. [26] compared the distance and similarity measures between intuitionistic fuzzy sets and applied them in a pattern recognition problem. 
Recently, Luo and Zhao [24] developed a new distance measure between intuitionistic fuzzy sets based on a matrix norm and a strictly increasing (or decreasing) binary function. Also, Garg and Kaur [13] introduced some new distance measures for cubic intuitionistic fuzzy sets which characterize the membership and non-membership in the form of interval-valued IFS.

Zhang and $\mathrm{Xu}$ [44] studied a distance measure of Pythagorean fuzzy numbers and used it in multi-criteria decision making by extending the TOPSIS method. Next, Li and Zeng [23] proposed a new distance measure for PFSs and Pythagorean fuzzy numbers considering four parameters i.e., membership degree, nonmembership degree, strength of commitment about membership and direction of commitment.

Cuong [6] stuied the some properties of picture fuzzy sets and discussed few distance measures between them. Son [27] developed a generalized picture distance measure and integrate it to a novel hierarchical picture fuzzy clustering method called hierarchical picture clustering. Thong et al. [31,32] applied a novel picture fuzzy clustering techniques for complex data and particle swarm optimization. Wei [34] studied a technique in light of the picture fuzzy weighted cross-entropy and applied it in the multi attribute decision making problem to rank the alternatives under consideration. Also, Son [28] studied the picture association measures and applied in group decision making. Further, Dutta [8] modified the Son's distance measure and then find the solution of medical diagnosis problem by utilizing the proposed distance measure. Dinh et al. [7] proposed the distance measure and dissimilarity measure to measure the difference between two picture fuzzy sets. Wei and Hao [37] introduced the generalized dice similarity measure for picture fuzzy set and studied and applications. Next, Wei [36] discussed some similarity measures to quantify the similarity between picture fuzzy sets. Some new aggregation operators for picture fuzzy sets have been studied for solving different variety of problems, viz. Hamachar aggregation approach for assessing the performance of the best enterprise [20], Muirhead mean aggregation approach in evaluating the risk in the financial investment [33], Dombi aggregation operator in an multi-attribute decision making process [21]. Recently, Khalil et al. [22] developed some fundamental operations for interval-valued picture fuzzy set/picture fuzzy soft set with various properties and applied them to solve the decisionmaking problems. Zeng et al. [43] studied a novel picture fuzzy discriminant measure based on Jensen's inequality/exponential entropy in a probabilistic framework with important properties and illustrative examples.

Feng et al. [10] presented different lexicographic orders for intuitionistic fuzzy setup and also studied relationship between them. Feng et al. [9] provided a new perspective of intuitionistic fuzzy soft sets (IFSSs) along with its various standard binary operations. An algorithm to solve the decision-making problem has been provided and implemented by considering a case study related to a faculty appointment problem. Further, a modified preference ranking organization method for enrichment evaluation (PROMETHEE) based on IFSSs by considering some new notions of matrices such as deviation matrices, membership (or non-membership) preference matrices and aggregated intuitionistic fuzzy preference matrices to solve a multiple attribute decision-making problem have been duly discussed by Feng et al. [11]. Also, the Minkowski score functions for intuitionistic fuzzy values and also studied its different properties and presented an algorithm to solve decisionmaking problem based on IFSSs by utilizing the proposed score function have been studied by Feng et al. [12]. Yager [39] introduced the a class of nonstandard Pythagorean fuzzy subsets along with its various aggregation operators. Further, Yager [40] introduced a general class of fuzzy sets and called its as $q$-rung orthopair fuzzy sets in which the sum of the $q^{\text {th }}$ power of the membership grade and the $q^{\text {th }}$ power of the non-membership grades is less than equal to 1. Guleria and Bajaj [14] proposed a new notion of matrix called as Pythagorean fuzzy soft matrix (PFSM) along with their standard binary operations. Based on these PFSM, Bajaj and Guleria [3] provided a new dimensionality reduction 
technique and algorithm has also been devised to solve the decision making problem. In order to deal with the uncertainty, the concept of Pythagorean fuzzy set has been utilized in various other capacities also $[15,16]$.

The application of the distance measure is for depicting the divergence between the elements while the similarity measure provides the degree of coincidence. In literature, the concept of similarity measures is almost synchronous with the concept of the distance measures. Most of the proposed definitions of measures contain the three parameters (membership, nonmembership, and hesitancy) of uncertainty, which are somewhat promising to deal with the loss of information. Still we need to cater the necessity to promote the accuracy/rationality in the decision-making by incorporating the four parameters of uncertainty in a probabilistic framework.

The motivation behind proposing the novel concept in this paper is to consider the probabilistic occurrence of the membership \& non-occurrence of the non-membership value of the imprecise information along with two more parameters of indeterminacy and refusal. Hence, the proposed distance measure for the picture fuzzy sets incorporates the concept of probability along with the existing four parameters, i.e., the degree of membership, neutral membership, degree of non-membership and degree of refusal. This, which certainly widens the scope of coverage of the incomplete information and the uncertainty space. Since the proposed distance measure is of probabilistic nature in a composite form, therefore, it has different kinds of prospects in different practical applications where the uncertainty is in a probabilistic and fuzzified way. Additionally, we are proposing an algorithm for solving the pattern recognition problems/classification problems based on the proposed probabilistic distance measure which enhance the literature in an advantageous way.

The rest of the paper is organized as follows. The basic and fundamental definitions are being discussed in Section 2 for a ready reference to preliminaries. The proposed composite probabilistic distance measure for the picture fuzzy sets with its normalized form has been developed in Section 3. Based on the proposed distance measure, the algorithm for solving classification problems and decision making problems has been presented along with appropriate illustrative examples in Section 4. The comparative analysis of the obtained results over the existing methods for the illustrative examples and the advantages of composing the probabilistic randomness and refusal uncertainty in the proposed method have been studied in Section 5. Finally, the paper has been concluded in Section 6 stating the outlines of the scope for future work.

\section{Preliminaries}

In this section, we recall and present some fundamental concepts related to the picture fuzzy sets and their existing distance measures which are well known in literature. The generalization process from intuitionistic fuzzy sets to picture fuzzy sets may be well understood through the following definitions:

Let $U$ be the universe of discourse with $\mu_{M}: U \rightarrow[0,1]$ and $\nu_{M}: U \rightarrow[0,1]$ being the degree of membership and degree of non-membership respectively. The set $M=$ $\left\{<\alpha, \mu_{M}(\alpha), \nu_{M}(\alpha)>\mid \alpha \in U\right\}$ is called

- Intuitionistic fuzzy set $[1]$ in $U$ if it satisfies the condition $0 \leq \mu_{M}(\alpha)+\nu_{M}(\alpha) \leq$ 1 with the degree of indeterminacy given by

$$
\pi_{M}(\alpha)=1-\mu_{M}(\alpha)-\nu_{M}(\alpha) .
$$

- Pythagorean fuzzy set [38] or Intuitionistic fuzzy set of second type [2] in $U$ if it satisfies the condition $0 \leq \mu_{M}^{2}(\alpha)+\nu_{M}^{2}(\alpha) \leq 1$ with the degree of indeterminacy given by $\pi_{M}(\alpha)=\sqrt{1-\mu_{M}^{2}(\alpha)-\nu_{M}^{2}(\alpha)}$. 
The first ever probabilistic approach for intuitionistic fuzzy event was developed by Szmidt and Kacprzyk [29], which was an extension of Zadeh's fuzzy event/probability measure [42], given by

Definition 2.1. [29] Suppose the probability of occurrence of a intuitionistic fuzzy event $M \subset U=\left\{\alpha_{1}, \alpha_{2}, \ldots, \alpha_{n}\right\}$ be $p\left(\alpha_{1}\right), p\left(\alpha_{2}\right), \ldots, p\left(\alpha_{n}\right)$ respectively. Then the minimal probability $p_{\min }(M)$ and maximal probability $p_{\max }(M)$ of an intuitionistic fuzzy event $M$ is given as:

and

$$
p_{\min }(M)=\sum_{i=1}^{n} p\left(\alpha_{i}\right) \mu_{M}\left(\alpha_{i}\right)
$$

$$
p_{\max }(M)=p_{\min }(M)+\sum_{i=1}^{n} p\left(\alpha_{i}\right) \pi_{M}\left(\alpha_{i}\right) .
$$

Hence the probability of event $M$ is a number from the interval $\left[p_{\min }(M), p_{\max }(M)\right]$.

In order to have further generalization, we consider the universe of discourse $U$ with $\mu_{M}: U \rightarrow[0,1], \eta_{M}: U \rightarrow[0,1]$ and $\nu_{M}: U \rightarrow[0,1]$ being the degree of membership, degree of neutral membership (abstain) and degree of non-membership respectively. The set $M=\left\{\left\langle\alpha, \mu_{M}(\alpha), \eta_{M}(\alpha), \nu_{M}(\alpha)>\right| \alpha \in U\right\}$ is called as Picture fuzzy set [5] in $U$ if it satisfies the condition $\mu_{M}(\alpha)+\eta_{M}(\alpha)+\nu_{M}(\alpha) \leq 1$ with the degree of refusal given by

$$
r_{M}(\alpha)=1-\left[\mu_{M}(\alpha)+\eta_{M}(\alpha)+\nu_{M}(\alpha)\right] .
$$

We are presenting definitions of some important existing distance measures for picture fuzzy sets which are well known in literature.

Definition 2.2. [5] Suppose $M$ and $N$ are two picture fuzzy sets over $U$. Then the Hamming Distance between $M$ and $N$ is given by

$$
d_{p}(M, N)=\sum_{i=1}^{n}\left(\left|\mu_{M}\left(\alpha_{i}\right)-\mu_{N}\left(\alpha_{i}\right)\right|+\left|\eta_{M}\left(\alpha_{i}\right)-\eta_{N}\left(\alpha_{i}\right)\right|+\left|\nu_{M}\left(\alpha_{i}\right)-\nu_{N}\left(\alpha_{i}\right)\right|\right) .
$$

Subsequently, the Normalized Hamming Distance between $M$ and $N$ is given by

$$
d_{p}(M, N)=\frac{1}{n} \sum_{i=1}^{n}\left(\left|\mu_{M}\left(\alpha_{i}\right)-\mu_{N}\left(\alpha_{i}\right)\right|+\left|\eta_{M}\left(\alpha_{i}\right)-\eta_{N}\left(\alpha_{i}\right)\right|+\left|\nu_{M}\left(\alpha_{i}\right)-\nu_{N}\left(\alpha_{i}\right)\right|\right) .
$$

Definition 2.3. [5] Suppose $M$ and $N$ are two picture fuzzy sets over $U$. Then the Euclidean Distance between $M$ and $N$ is given by

$$
e_{p}(M, N)=\sqrt{\frac{1}{2} \sum_{i=1}^{n}\left(\mu_{M}\left(\alpha_{i}\right)-\mu_{N}\left(\alpha_{i}\right)\right)^{2}+\left(\eta_{M}\left(\alpha_{i}\right)-\eta_{N}\left(\alpha_{i}\right)\right)^{2}+\left(\nu_{M}\left(\alpha_{i}\right)-\nu_{N}\left(\alpha_{i}\right)\right)^{2}} .
$$

Subsequently, the Normalized Euclidean Distance between the picture fuzzy sets $M$ and $N$ is given by

$$
q_{p}(M, N)=\sqrt{\frac{1}{2 n} \sum_{i=1}^{n}\left(\mu_{M}\left(\alpha_{i}\right)-\mu_{N}\left(\alpha_{i}\right)\right)^{2}+\left(\eta_{M}\left(\alpha_{i}\right)-\eta_{N}\left(\alpha_{i}\right)\right)^{2}+\left(\nu_{M}\left(\alpha_{i}\right)-\nu_{N}\left(\alpha_{i}\right)\right)^{2}} .
$$

Recently, some generalized picture distance measures have been proposed $[7,27]$ with possible applications.

Definition 2.4. [27] Suppose $M$ and $N$ are two picture fuzzy sets over $U$. Then the generalized picture distance measure between $M$ and $N$ is given by

$$
d_{G}(M, N)=\frac{\left(\frac{1}{n} \sum_{i=1}^{n}\left(\frac{\Delta \mu_{i}^{p}+\Delta \eta_{i}^{p}+\Delta \mu_{i}^{p}}{3}\right)+\max \left\{\Delta \mu_{i}^{p}, \Delta \eta_{i}^{p}, \Delta \mu_{i}^{p}\right\}\right)^{\frac{1}{p}}}{\left(\frac{1}{n} \sum_{i=1}^{n}\left(\frac{\Delta \mu_{i}^{p}+\Delta \eta_{i}^{p}+\Delta \mu_{i}^{p}}{3}\right)+\max \left\{\Delta \mu_{i}^{p}, \Delta \eta_{i}^{p}, \Delta \mu_{i}^{p}\right\}\right)^{\frac{1}{p}}+\left(\max _{i}\left\{\Phi_{i}^{M}, \Phi_{i}^{N}\right\}+\frac{1}{n} \sum_{i=1} n\left|\Phi_{i}^{M}-\Phi_{i}^{N}\right|^{p}\right)^{\frac{1}{p}}+1} ;
$$


where $\Delta \mu_{i}=\left|\mu_{M}\left(\alpha_{i}\right)-\mu_{N}\left(\alpha_{i}\right)\right|, \Delta \eta_{i}=\left|\eta_{M}\left(\alpha_{i}\right)-\eta_{N}\left(\alpha_{i}\right)\right|, \Delta \nu_{i}=\left|\nu_{M}\left(\alpha_{i}\right)-\nu_{N}\left(\alpha_{i}\right)\right|$, $\Phi_{i}^{M}=\left|\mu_{M}\left(\alpha_{i}\right)+\eta_{M}\left(\alpha_{i}\right)+\nu_{M}\left(\alpha_{i}\right)\right|$ and $\Phi_{i}^{N}=\left|\mu_{N}\left(\alpha_{i}\right)+\eta_{N}\left(\alpha_{i}\right)+\nu_{N}\left(\alpha_{i}\right)\right|$.

Definition 2.5. [7] Suppose $M$ and $N$ are two picture fuzzy sets over $U$. Then the some of distance measures between $M$ and $N$ are given by

$$
\begin{gathered}
D_{H}(M, N)=\frac{1}{3 n} \sum_{i=1}^{n}\left(\left|\mu_{M}\left(\alpha_{i}\right)-\mu_{N}\left(\alpha_{i}\right)\right|+\left|\eta_{M}\left(\alpha_{i}\right)-\eta_{N}\left(\alpha_{i}\right)\right|+\left|\nu_{M}\left(\alpha_{i}\right)-\nu_{N}\left(\alpha_{i}\right)\right|\right) ; \\
D_{E}(M, N)=\left(\sum_{i=1}^{n}\left(\mu_{M}\left(\alpha_{i}\right)-\mu_{N}\left(\alpha_{i}\right)\right)^{2}+\left(\eta_{M}\left(\alpha_{i}\right)-\eta_{N}\left(\alpha_{i}\right)\right)^{2}+\left(\nu_{M}\left(\alpha_{i}\right)-\nu_{N}\left(\alpha_{i}\right)\right)^{2}\right)^{\frac{1}{2}} \\
D_{H}^{m}(M, N)=\frac{1}{n} \sum_{i=1}^{n} \max \left(\left|\mu_{M}\left(\alpha_{i}\right)-\mu_{N}\left(\alpha_{i}\right)\right|,\left|\eta_{M}\left(\alpha_{i}\right)-\eta_{N}\left(\alpha_{i}\right)\right|,\left|\nu_{M}\left(\alpha_{i}\right)-\nu_{N}\left(\alpha_{i}\right)\right|\right) ; \\
D_{E}^{m}(M, N)=\left(\sum_{i=1}^{n} \max \left(\left(\mu_{M}\left(\alpha_{i}\right)-\mu_{N}\left(\alpha_{i}\right)\right)^{2},\left(\eta_{M}\left(\alpha_{i}\right)-\eta_{N}\left(\alpha_{i}\right)\right)^{2},\left(\nu_{M}\left(\alpha_{i}\right)-\nu_{N}\left(\alpha_{i}\right)\right)^{2}\right)\right)^{\frac{1}{2}} .
\end{gathered}
$$

\section{Probabilistic distance measure for picture fuzzy sets}

In this section, we develop a novel concept to address the probabilistic randomness with picture fuzzy uncertainty in a composite way and propose a probabilistic distance measure for the picture fuzzy sets. For this purpose, we first present a new definition for probability of occurrence and non-occurrence of a picture fuzzy event.

Definition 3.1. Let $U$ be the universe of discourse/sample space with $M=\left\{\alpha_{1}, \alpha_{2}, \ldots, \alpha_{n}\right\}$ as a set of picture fuzzy events. Consider the probability of occurrence of the picture fuzzy events as $p\left(\alpha_{1}\right), p\left(\alpha_{2}\right), \ldots, p\left(\alpha_{n}\right)$ respectively. Then, the minimal probability of occurrence $p_{\min }(M)$ and maximal probability of occurrence $p_{\max }(M)$ of the set of picture fuzzy events $M$ is given by:

$$
p_{\min }(M)=\sum_{i=1}^{n} p\left(\alpha_{i}\right) \mu_{M}\left(\alpha_{i}\right)
$$

and

$$
p_{\max }(M)=p_{\min }(M)+\sum_{i=1}^{n} p\left(\alpha_{i}\right) \eta_{M}\left(\alpha_{i}\right)
$$

Hence, the probability of occurrence of the picture fuzzy event $M$, denoted by $p(M)$, is a number lying in the interval $\left[p_{\min }(M), p_{\max }(M)\right]$, that is,

$$
p(M) \in\left[p_{\min }(M), p_{\max }(M)\right] .
$$

Consequently, suppose that the probability of non-occurrence of the picture fuzzy events as $q\left(\alpha_{1}\right), q\left(\alpha_{2}\right), \ldots, q\left(\alpha_{n}\right)$ respectively. Then, the minimal probability of non-occurrence $q_{\min }(M)$ and maximal probability of non-occurrence $q_{\max }(M)$ of the set of picture fuzzy events $M$ is given by:

$$
q_{\min }(M)=\sum_{i=1}^{n} q\left(\alpha_{i}\right) \nu_{M}\left(\alpha_{i}\right)
$$

and

$$
q_{\max }(M)=q_{\min }(M)+\sum_{i=1}^{n} q\left(\alpha_{i}\right) r_{M}\left(\alpha_{i}\right) .
$$

Hence, the probability of non-occurrence of the picture fuzzy event $M$, denoted by $q(M)$ is a number lying in the interval $\left[q_{\min }(M), q_{\max }(M)\right]$, i.e.,

$$
q(M) \in\left[q_{\min }(M), q_{\max }(M)\right] .
$$


Example 3.1: Consider $U$ as the universe of discourse/sample space and $M=\left\{\left(\alpha_{1}, 0.46,0.36,0.1\right),\left(\alpha_{2}, 0.73,0.04,0.11\right),\left(\alpha_{3}, 0.48,0.26,0.16\right)\right\}$ as a set of picture fuzzy events. Suppose the probability of occurrence of the picture fuzzy events is given by $p\left(\alpha_{1}\right)=0.02717, p\left(\alpha_{2}\right)=0.6956, p\left(\alpha_{n}\right)=0.2173$ and the probability of non-occurrence of the picture fuzzy events is given by

$$
q\left(\alpha_{1}\right)=0.5714, q\left(\alpha_{2}\right)=0.0714, q\left(\alpha_{n}\right)=0.2857 .
$$

Then, in view of Definition 3.1, we have

$$
p_{\min }(M)=0.02717 \times 0.46+0.6956 \times 0.36+0.2173 \times 0.1=0.62459 ;
$$

and

$$
p_{\max }(M)=0.62459+(0.02717 \times 0.36+0.6956 \times 0.04+0.2173 \times 0.26)=0.71869 .
$$

Hence, $p(M) \in[0.62459,0.71869]$. On similar lines, we obtain $q_{\min }(M)=0.11071$ and $q_{\max }(M)=0.19356$. Thus, $q(M) \in[0.11071,0.19356]$.

Remark: It may be noted that the values of $p(M)$ and $q(M)$ are equally likely in their respective intervals. Without loss of generality, it will be always wise to choose the mean of the intervals in order to avoid any kind of left/right deviations in the selection.

The origin of the proposed novel distance measure between picture fuzzy sets is because of inter-connecting and incorporating the concept of probabilistic randomness with the existing four fuzziness parameters, that is, the degree of membership, neutral membership, degree of non-membership and degree of refusal, which may be well understood with the help of the following structure of the problem of categorization:

\section{Problem outline and proposed solution structure:}

Suppose a person wants to classify an unknown pattern among the different known $m$ classes provided by the different $k$ experts, (say $e_{k}^{\prime} s$ ) by utilizing a distance measure. We evaluate the distance of the unknown pattern with the each known class and make a decision in favor of that class which has the minimum value of the computed distance measure with the unknown pattern.

Let $U=\left\{\alpha_{1}, \alpha_{2}, \ldots, \alpha_{n}\right\}$ be the universe of discourse and consider the opinion of the expert regarding each $\alpha_{i}$, given in the form of picture fuzzy set as:

$$
M=\left\{\left(\alpha_{i}, \mu_{M}\left(\alpha_{i}\right), \eta_{M}\left(\alpha_{i}\right), \nu_{M}\left(\alpha_{i}\right)\right) \mid \alpha_{i} \in X\right\} .
$$

Consider $p_{i}$ as the probability of the useful information in the opinion of expert with respect to the degree of membership. If there is no loss of the information in membership value then the value of the degree of membership is absolutely correct. Also, consider $q_{i}$ as the probability of the useful information in the opinion of expert with respect to the degree of non-membership. If there is no loss of the information in non-membership value then the value of the degree of non-membership is absolutely correct. Analogously, $s_{i}$ be the probability of the useful information in the opinion of expert with respect to abstain/ neutral membership. If there is no loss of the information in neutral membership value then the value of the degree of neutral membership is absolutely correct. Now, we have to find the value of $p_{i}, q_{i}$ and $s_{i}$ corresponding to the given data.

Since, the degree of refusal $r_{M}\left(\alpha_{i}\right)=1-\left(\mu_{M}\left(\alpha_{i}\right)+\eta_{M}\left(\alpha_{i}\right)+\nu_{M}\left(\alpha_{i}\right)\right)$, therefore $r_{M}\left(\alpha_{i}\right)$ is correct if all other parameters are simultaneously correct. Also, we have made assumption that the degree of membership, degree of neutral membership and degree of non-membership are correct with chances $p_{i}, q_{i}$ and $s_{i}$, respectively and are independent. Thus, the probability of the event that all the three parameters - degree of membership, degree of neutral membership and degree of non-membership, are simultaneously correct 
is $p_{i} q_{i} s_{i}$. Hence, the absolute refusal part $r_{M}^{\prime}\left(\alpha_{i}\right)=p_{i} q_{i} s_{i} r_{M}\left(\alpha_{i}\right)$. So, the absolutely correct membership $\mu_{M}^{\prime}\left(\alpha_{i}\right)$, neutral membership $\eta_{M}^{\prime}\left(\alpha_{i}\right)$ and non-membership $\nu_{M}^{\prime}\left(\alpha_{i}\right)$ are as follows:

$$
\begin{aligned}
\mu_{M}^{\prime}\left(\alpha_{i}\right) & =p_{i} \mu_{M}\left(\alpha_{i}\right) \leq \mu_{M}\left(\alpha_{i}\right) ; \\
\eta_{M}^{\prime}\left(\alpha_{i}\right) & =q_{i} \eta_{M}\left(\alpha_{i}\right) \leq \eta_{M}\left(\alpha_{i}\right) ; \\
\nu_{M}^{\prime}\left(\alpha_{i}\right) & =s_{i} \nu_{M}\left(\alpha_{i}\right) \leq \nu_{M}\left(\alpha_{i}\right) .
\end{aligned}
$$

Now, in each part, i.e., $\mu_{M}^{\prime}, \eta_{M}^{\prime}$ and $\nu_{M}^{\prime}$, there is some loss form the original values $\mu_{M}, \eta_{M}$, $\nu_{M}$. Therefore, we add total loss in the refusal part and define a new picture fuzzy set as $M=\left\{\left(\alpha_{i}, \mu_{M}^{\prime}\left(\alpha_{i}\right), \eta_{M}^{\prime}\left(\alpha_{i}\right), \nu_{M}^{\prime}\left(\alpha_{i}\right)\right) \mid \alpha_{i} \in X\right\}$, where

$$
r_{M}^{\prime}\left(\alpha_{i}\right)=p_{i} q_{i} s_{i} r_{M}\left(\alpha_{i}\right)+\text { loss, }
$$

and

$$
\text { loss }=1-\left(p_{i} \mu_{M}\left(\alpha_{i}\right)+q_{i} \eta_{M}\left(\alpha_{i}\right)+s_{i} \nu_{M}\left(\alpha_{i}\right)+p_{i} q_{i} s_{i} r_{M}\left(\alpha_{i}\right)\right) .
$$

If the uncertainty involved in the fuzzy variable is absolutely correct then there is no loss of the above stated parameters, otherwise some loss is always there. From the definition of picture fuzzy set, we have

$$
\mu_{M}^{\prime}\left(\alpha_{i}\right)+\eta_{M}^{\prime}\left(\alpha_{i}\right)+\nu_{M}^{\prime}\left(\alpha_{i}\right)+r_{M}^{\prime}\left(\alpha_{i}\right)=1
$$

which implies

$$
p_{i} \mu_{M}\left(\alpha_{i}\right)+q_{i} \eta_{M}\left(\alpha_{i}\right)+s_{i} \nu_{M}\left(\alpha_{i}\right)+p_{i} q_{i} s_{i} r_{M}^{\prime}\left(\alpha_{i}\right) \leq 1
$$

Now,

$$
p_{i} \mu_{M}\left(\alpha_{i}\right)+q_{i} \eta_{M}\left(\alpha_{i}\right)+s_{i} \nu_{M}\left(\alpha_{i}\right)+p_{i} q_{i} s_{i} r_{M}^{\prime}\left(\alpha_{i}\right) \leq p_{i}+q_{i}+s_{i}+p_{i} q_{i} s_{i} .
$$

For the different possible combinations of $\left(p_{i}, q_{i}, s_{i}\right)$ and $\left(\mu_{M}\left(\alpha_{i}\right), \eta_{M}\left(\alpha_{i}\right), \nu_{M}\left(\alpha_{i}\right), r_{M}\left(\alpha_{i}\right)\right.$, the L.H.S of equation (3.2) may approach to its maximum value, i.e., close to 1 , which implies that

$$
p_{i}+q_{i}+s_{i}+p_{i} q_{i} s_{i} \geq 1
$$

Suppose for each $\alpha_{i}$, if an expert assigns absolutely correct values to all the three parameters, then we have $p_{i}+q_{i}+s_{i}+p_{i} q_{i} s_{i}=4$, otherwise

$$
p_{i}+q_{i}+s_{i}+p_{i} q_{i} s_{i}<4 .
$$

Therefore, from equation (3.3) and (3.4), we have

$$
1 \leq p_{i}+q_{i}+s_{i}+p_{i} q_{i} s_{i} \leq 4
$$

Case 1 : If there is no loss in the information, i.e., $p_{i}=1, q_{i}=1$ and $s_{i}=1$, then $p_{i}+q_{i}+s_{i}+p_{i} q_{i} s_{i}=4$.

Case $2: p_{i} \neq 1, q_{i} \neq 1$ and $s_{i} \neq 1$. As $p_{i}+q_{i}+s_{i}+p_{i} q_{i} s_{i}$ decreases then at least one of the $p_{i}$ or $q_{i}$ or $s_{i}$ decreases, which means loss of information takes place.

Let $p_{i}, q_{i}, s_{i}$ be such that $p_{i}+q_{i}+s_{i}+p_{i} q_{i} s_{i}=\beta<4$, which implies

$$
s_{i}=\frac{\beta-p_{i}-q_{i}}{1-p_{i} q_{i}} .
$$

In order to establish the feasibility of the solution of the problem, we would select $0 \leq$ $p_{i} \leq 1$ and $0 \leq q_{i} \leq 1$ from the data so that $0 \leq s_{i} \leq 1$. Thus, after selecting such $p_{i}, q_{i}$, it may be noted that $s_{i}$ decreases as $\beta$ decreases and $s_{i}$ takes the least value when $\beta=1$, i.e.,

$$
s_{i}=\frac{1-p_{i}-q_{i}}{1-p_{i} q_{i}} .
$$


Now, in view of the maximum possible loss in the information and taking all the four fuzzy parameters into account, we define a composite probabilistic distance measure for picture fuzzy sets $M$ and $N$, denoted by $d_{P}(M, N)$, given by

$$
\begin{aligned}
d_{P}(M, N) & =\left(\frac{1}{2} \sum_{i=1}^{n} p_{i}\left(\mu_{M}\left(\alpha_{i}\right)-\mu_{N}\left(\alpha_{i}\right)\right)^{2}+\frac{1-p_{i}-q_{i}}{1-p_{i} q_{i}}\left(\eta_{M}\left(\alpha_{i}\right)-\eta_{N}\left(\alpha_{i}\right)\right)^{2}\right. \\
& \left.+q_{i}\left(\nu_{M}\left(\alpha_{i}\right)-\nu_{N}\left(\alpha_{i}\right)\right)^{2}+\left(p_{i} q_{i}\right)\left(\frac{1-p_{i}-q_{i}}{1-p_{i} q_{i}}\right)\left(r_{M}\left(\alpha_{i}\right)-r_{N}\left(\alpha_{i}\right)\right)^{2}\right)^{\frac{1}{2}} .
\end{aligned}
$$

Subsequently, the normalized form of the above defined distance measure can be written as follows:

$$
\begin{aligned}
d_{P}^{N o r}(M, N) & =\left(\frac{1}{2 n} \sum_{i=1}^{n} p_{i}\left(\mu_{M}\left(\alpha_{i}\right)-\mu_{N}\left(\alpha_{i}\right)\right)^{2}+\frac{1-p_{i}-q_{i}}{1-p_{i} q_{i}}\left(\eta_{M}\left(\alpha_{i}\right)-\eta_{N}\left(\alpha_{i}\right)\right)^{2}\right. \\
& \left.+q_{i}\left(\nu_{M}\left(\alpha_{i}\right)-\nu_{N}\left(\alpha_{i}\right)\right)^{2}+\left(p_{i} q_{i}\right)\left(\frac{1-p_{i}-q_{i}}{1-p_{i} q_{i}}\right)\left(r_{M}\left(\alpha_{i}\right)-r_{N}\left(\alpha_{i}\right)\right)^{2}\right)^{\frac{1}{2}} .
\end{aligned}
$$

Remark: It may be noted that the proposed distance measures satisfy the following conditions of a pseudo-metric on the universe of discourse:

- $C_{1}: 0 \leq d_{P}(M, N) \leq 1$,

- $C_{2}: d_{P}(M, N)=0 \Leftrightarrow M=N$,

- $C_{3}: d_{P}(M, N)=d_{P}(N, M)$.

Theorem 3.2. The distance measure $d_{P}(M, N)$ between two arbitrary picture fuzzy sets $M$ and $N$ over the universe of discourse $U$ satisfy the conditions of a pseudo-metric.

Proof: Let $M$ and $N$ be two arbitrary picture fuzzy sets over the universe of discourse $U$. In order to show that the distance measure $d_{P}(M, N)$ given by equation (3.6) satisfies the conditions of a pseudo-metric, we need to validate the conditions $C_{1}, C_{2}$ and $C_{3}$ stated in the above remark.

- Condition $C_{1}$ : By equation 3.6, we certainly have $d_{P}(M, N) \geq 0$ as it is the sum of only non-negative terms. Hence, it remains to show that $d_{P}(M, N) \leq 1$.

For the picture fuzzy sets $M$ and $N$ in probabilistic framework, we have

$0 \leq \mu_{M}\left(\alpha_{i}\right), \mu_{N}\left(\alpha_{i}\right), \eta_{M}\left(\alpha_{i}\right), \eta_{N}\left(\alpha_{i}\right), \nu_{M}\left(\alpha_{i}\right), \nu_{N}\left(\alpha_{i}\right), r_{M}\left(\alpha_{i}\right), r_{N}\left(\alpha_{i}\right), p_{i}, q_{i} \leq 1$.

Hence, this implies that

$$
\begin{gathered}
0 \leq\left(\mu_{M}\left(\alpha_{i}\right)-\mu_{N}\left(\alpha_{i}\right)\right)^{2} \leq 1,0 \leq\left(\eta_{M}\left(\alpha_{i}\right)-\eta_{N}\left(\alpha_{i}\right)\right)^{2} \leq 1, \\
0 \leq\left(\nu_{M}\left(\alpha_{i}\right)-\nu_{N}\left(\alpha_{i}\right)\right)^{2} \leq 1,0 \leq\left(r_{M}\left(\alpha_{i}\right)-r_{N}\left(\alpha_{i}\right)\right)^{2} \leq 1, \\
0 \leq \frac{1-p_{i}-q_{i}}{1-p_{i} q_{i}} \leq 1 \text { and } 0 \leq p_{i} q_{i}\left(\frac{1-p_{i}-q_{i}}{1-p_{i} q_{i}}\right) \leq 1 .
\end{gathered}
$$

Therefore,

$$
\begin{aligned}
& 0 \leq\left(\frac{1}{2} \sum_{i=1}^{n} p_{i}\left(\mu_{M}\left(\alpha_{i}\right)-\mu_{N}\left(\alpha_{i}\right)\right)^{2}+\frac{1-p_{i}-q_{i}}{1-p_{i} q_{i}}\left(\eta_{M}\left(\alpha_{i}\right)-\eta_{N}\left(\alpha_{i}\right)\right)^{2}\right. \\
& \left.+q_{i}\left(\nu_{M}\left(\alpha_{i}\right)-\nu_{N}\left(\alpha_{i}\right)\right)^{2}+\left(p_{i} q_{i}\right)\left(\frac{1-p_{i}-q_{i}}{1-p_{i} q_{i}}\right)\left(r_{M}\left(\alpha_{i}\right)-r_{N}\left(\alpha_{i}\right)\right)^{2}\right)^{\frac{1}{2}} \leq 1 ;
\end{aligned}
$$

which implies that $d_{P}(M, N) \leq 1$. 
- Condition $C_{2}$ : For any two picture fuzzy sets $M$ and $N, d_{P}(M, N)=0$ implies that

$$
\begin{aligned}
& \left(\frac{1}{2 n} \sum_{i=1}^{n} p_{i}\left(\mu_{M}\left(\alpha_{i}\right)-\mu_{N}\left(\alpha_{i}\right)\right)^{2}+\frac{1-p_{i}-q_{i}}{1-p_{i} q_{i}}\left(\eta_{M}\left(\alpha_{i}\right)-\eta_{N}\left(\alpha_{i}\right)\right)^{2}\right. \\
& \left.+q_{i}\left(\nu_{M}\left(\alpha_{i}\right)-\nu_{N}\left(\alpha_{i}\right)\right)^{2}+\left(p_{i} q_{i}\right)\left(\frac{1-p_{i}-q_{i}}{1-p_{i} q_{i}}\right)\left(r_{M}\left(\alpha_{i}\right)-r_{N}\left(\alpha_{i}\right)\right)^{2}\right)^{\frac{1}{2}}=0, \forall i ; \\
& \Rightarrow\left(\mu_{M}\left(\alpha_{i}\right)-\mu_{N}\left(\alpha_{i}\right)\right)^{2}=0,\left(\eta_{M}\left(\alpha_{i}\right)-\eta_{N}\left(\alpha_{i}\right)\right)^{2}=0,\left(\nu_{M}\left(\alpha_{i}\right)-\nu_{N}\left(\alpha_{i}\right)\right)^{2}=0, \\
& \quad\left(r_{M}\left(\alpha_{i}\right)-r_{N}\left(\alpha_{i}\right)\right)^{2}=0, \forall i ; \\
& \Rightarrow \mu_{M}\left(\alpha_{i}\right)-\mu_{N}\left(\alpha_{i}\right)=0, \eta_{M}\left(\alpha_{i}\right)-\eta_{N}\left(\alpha_{i}\right)=0, \nu_{M}\left(\alpha_{i}\right)-\nu_{N}\left(\alpha_{i}\right)=0, \\
& \quad r_{M}\left(\alpha_{i}\right)-r_{N}\left(\alpha_{i}\right)=0, \forall i ; \\
& \Rightarrow \mu_{M}\left(\alpha_{i}\right)=\mu_{N}\left(\alpha_{i}\right), \eta_{M}\left(\alpha_{i}\right)=\eta_{N}\left(\alpha_{i}\right), \nu_{M}\left(\alpha_{i}\right)=\nu_{N}\left(\alpha_{i}\right), r_{M}\left(\alpha_{i}\right)=r_{N}\left(\alpha_{i}\right), \forall i ; \\
& \Rightarrow M=N .
\end{aligned}
$$

The other way implication may similarly be understood. Therefore,

$$
d_{P}(M, N)=0 \Leftrightarrow M=N .
$$

- Condition $C_{3}$ : In view of the equation (3.6) using the interchanging process, it is quite obvious that $d_{P}(M, N)=d_{P}(N, M)$.

Hence, the distance measure $d_{P}(M, N)$ satisfies all the conditions of a pseudo-metric.

\section{Algorithm and application based on proposed probabilistic measure}

In this section, we propose an algorithm for solving a general classification problem based on the proposed composite probabilistic distance measure for picture fuzzy sets. The classification problem consists of some number of standard known categorical classes with several types of features space associated with each class [36]. For the sake of wider applicability, we have also considered a decision making problem of financial investment risk under the enterprize environment.

For accomplishing the task of classification of an unknown data, we propose an algorithm with the help of the flow chart given in Figure 2.

The procedural steps of the proposed algorithm has been explicitly outlined as follows:

- Step 1 Determine the minimum membership value and non-membership value from the set of membership values \& non-membership values assigned by the experts $A_{j}(j=$ $1,2, \ldots, n)$ to the objects $x_{i}(i=1,2, \ldots, m)$, that is,

$$
\min _{j} \mu_{A_{j}}\left(x_{i}\right)=\mu^{\prime}\left(x_{i}\right) \text { and } \min _{j} \nu_{A_{j}}\left(x_{i}\right)=\nu^{\prime}\left(x_{i}\right) .
$$

- Step 2 Collect all the minimum membership values and non-membership values for each $x_{i}$ for $i=1,2, \ldots, m$ to form the set $E=\left\{\mu\left(x_{1}\right), \mu\left(x_{2}\right), \ldots, \mu\left(x_{m}\right)\right\}$ and $E^{\prime}=$ $\left\{\nu\left(x_{1}\right), \mu\left(x_{2}\right), \ldots, \mu\left(x_{m}\right)\right\}$.

- Step 3 We compute the sum $S=\sum_{i=1}^{m} \mu^{\prime}\left(x_{i}\right)$ and $S^{\prime}=\sum_{i=1}^{m} \nu^{\prime}\left(x_{i}\right)$ and determine the probability of occurrence $p\left(x_{i}\right)=\frac{\mu^{\prime}\left(x_{i}\right)}{S}$ and the probability of non-occurrence $q\left(x_{i}\right)=$ $\frac{\nu^{\prime}\left(x_{i}\right)}{S^{\prime}}$.

- Step 4 Compute the minimum probability of occurrence $p_{\min }\left(A_{j}\right)=\sum_{i=1}^{m} p\left(x_{i}\right) \mu_{A_{j}}\left(x_{i}\right)$ and the minimum probability of non-occurrence $q_{\min }\left(A_{j}\right)=\sum_{i=1}^{m} q\left(x_{i}\right) \nu_{A_{j}}\left(x_{i}\right)$. 


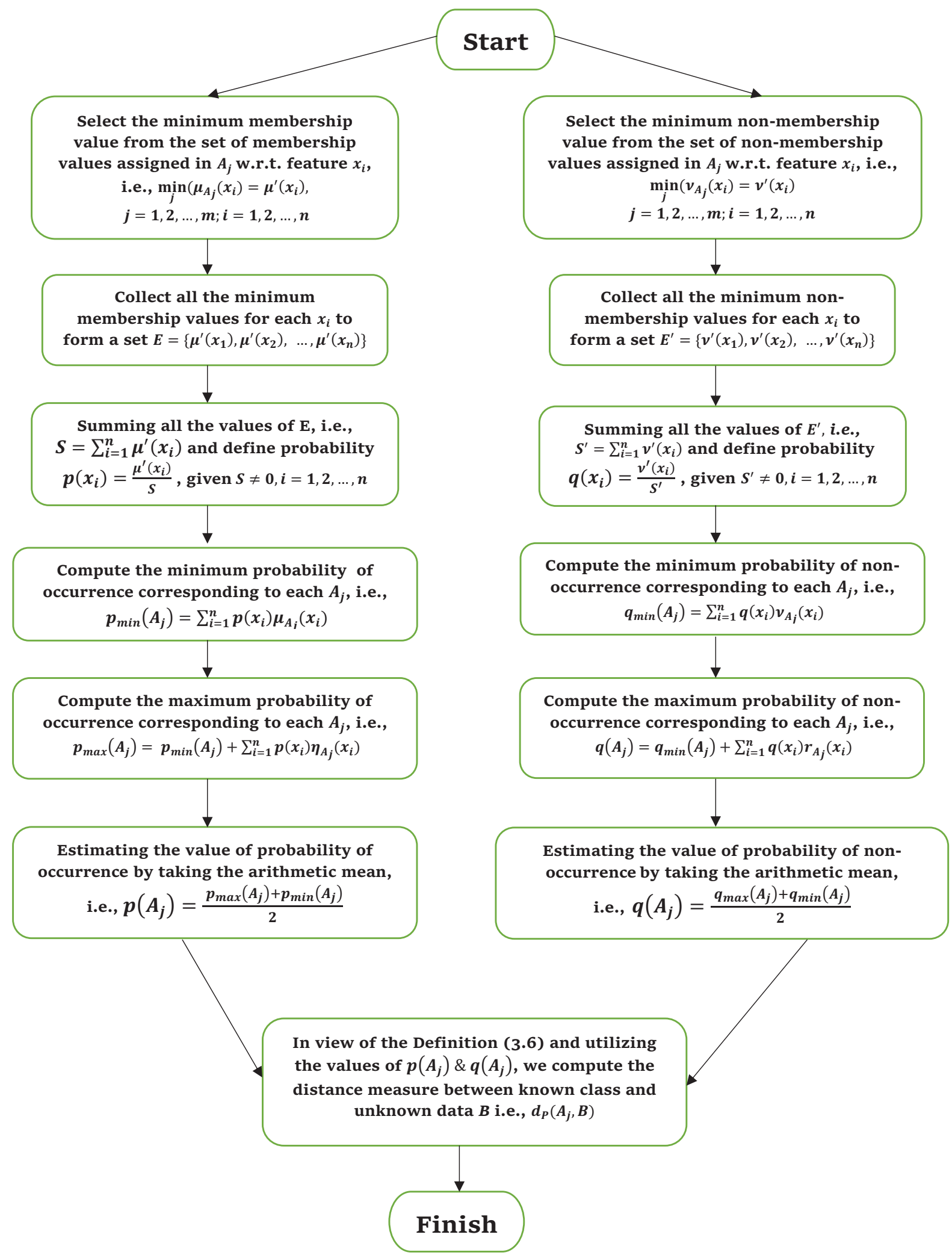

Figure 2. Flow Chart of the Proposed Algorithm 
- Step 5 Next, we compute the maximum probability of occurrence $p_{\max }\left(A_{j}\right)=p_{\min }\left(A_{j}\right)+$ $\sum_{i=1}^{m} p\left(x_{i}\right) \eta_{A_{j}}\left(x_{i}\right)$ and the maximum probability of non-occurrence $q_{\max }\left(A_{j}\right)=q_{\min }\left(A_{j}\right)+$ $\sum_{i=1}^{m} q\left(x_{i}\right) r_{A_{j}}\left(x_{i}\right)$

- Step 6 Find the value of probability of occurrence $p\left(A_{j}\right)=\frac{p_{\max }\left(A_{j}\right)+p_{\min }\left(A_{j}\right)}{2}$ and probability of non-occurrence $q\left(A_{j}\right)=\frac{q_{\max }\left(A_{j}\right)+q_{\min }\left(A_{j}\right)}{2}$.

- Step 7 Finally, by using the equation (3.6), we compute the determining probabilistic picture distance measures of all the known classes with respect to the unknown class and on the basis of the above obtained values of the distances, we conclude our desired result.

\subsection{Building material classification problem}

Here, we consider a particular example to demonstrate the building material recognition problem. This classification problem related to the building materials is based on the observations and expert opinions. We have considered 4 classes of the building material each represented by the picture fuzzy sets $A_{1}, A_{2}, A_{3}, A_{4}$. Also, we have taken 7 space features of material samples $X=\left\{x_{1}, x_{2}, x_{3}, x_{4}, x_{5}, x_{6}, x_{7}\right\}$ and an unknown material $B$, which is to be classified in one of the 4 known classes. The proposed algorithm has been implemented over a recent standard data problem (refer Wei [36]).

In such kind of classification problem, the aim is to identify the class of unknown building material using the degree of distance measure of unknown material with that of known building materials along with the incorporation of probability of occurence/nonoccurrence of fuzzy event. The briefed steps of algorithm for finding the class of unknown building material are described as:

- Obtain the information about the known building materials in the form of picture fuzzy sets.

- Applying the procedural steps of the proposed algorithm.

- Rank the distance measures to find out the class of unknown material.

Now, applying the procedural steps of the proposed algorithm over the above stated problem as follows:

- Step 1 We first determine the minimum membership value and non-membership value from the set of membership values \& non-membership values assigned by the experts $A_{j}(j=1,2, \ldots, 4)$ to the objects $x_{i}(i=1,2, \ldots, 7)$, that is,

$$
\min _{j} \mu_{A_{j}}\left(x_{i}\right)=\mu^{\prime}\left(x_{i}\right) \text { and } \min _{j} \nu_{A_{j}}\left(x_{i}\right)=\nu^{\prime}\left(x_{i}\right) .
$$

For example, here for $i=1$, we have $\min _{j=1}^{4} \mu_{A_{j}}\left(x_{1}\right)=0.17$ and $\min _{j=1}^{4} \nu_{A_{j}}\left(x_{1}\right)=0.0$.

- Step 2 Next, we collect all the minimum membership values and non-membership values for each $x_{i}$ for $i=1,2, \ldots, 7$ to form the set $E=\left\{\mu\left(x_{1}\right), \mu\left(x_{2}\right), \ldots, \mu\left(x_{7}\right)\right\}$ and $E^{\prime}=$ $\left\{\nu\left(x_{1}\right), \mu\left(x_{2}\right), \ldots, \mu\left(x_{7}\right)\right\}$ as follows:

$$
E=\{0.17,0.1,0.53,0.07,0.02,0.08,0.15\}
$$

and

$$
E^{\prime}=\{0.0,0.0,0.0,0.03,0.05,0.02,0.05\}
$$

- Step 3 We compute the sum $S=\sum_{i=1}^{7} \mu^{\prime}\left(x_{i}\right)=1.12$ and $S^{\prime}=\sum_{i=1}^{7} \nu^{\prime}\left(x_{i}\right)=0.15$ and determine the probability of occurrence $p\left(x_{i}\right)=\frac{\mu^{\prime}\left(x_{i}\right)}{S}$ and the probability of non-occurrence 
$q\left(x_{i}\right)=\frac{\nu^{\prime}\left(x_{i}\right)}{S^{\prime}}$ as follows:

$$
\begin{gathered}
p\left(x_{1}\right)=0.1518 ; p\left(x_{2}\right)=0.0893 ; p\left(x_{3}\right)=0.4732 ; p\left(x_{4}\right)=0.0625 ; \\
p\left(x_{5}\right)=0.0179 ; p\left(x_{6}\right)=0.0714 ; p\left(x_{7}\right)=0.1339 ;
\end{gathered}
$$

and

$$
\begin{array}{r}
q\left(x_{1}\right)=0.0 ; q\left(x_{2}\right)=0.0 ; q\left(x_{3}\right)=0.0 ; q\left(x_{4}\right)=0.2 ; \\
q\left(x_{5}\right)=0.333 ; q\left(x_{6}\right)=0.133 ; q\left(x_{7}\right)=0.333 .
\end{array}
$$

- Step 4 Next, we compute the minimum probability of occurrence $p_{\min }\left(A_{j}\right)=\sum_{i=1}^{7} p\left(x_{i}\right) \mu_{A_{j}}\left(x_{i}\right)$ i.e.,

$$
p_{\min }\left(A_{1}\right)=0.3986 ; p_{\min }\left(A_{2}\right)=0.7364 ; p_{\min }\left(A_{3}\right)=0.605 ; p_{\min }\left(A_{4}\right)=0.7171 ;
$$

and the minimum probability of non-occurrence $q_{\min }\left(A_{j}\right)=\sum_{i=1}^{7} q\left(x_{i}\right) \nu_{A_{j}}\left(x_{i}\right)$ i.e.,

$$
q_{\min }\left(A_{1}\right)=0.1087 ; q_{\min }\left(A_{2}\right)=0.1493 ; q_{\min }\left(A_{3}\right)=0.0747 ; q_{\min }\left(A_{4}\right)=0.0613 .
$$

- Step 5 Next, we compute the maximum probability of occurrence

$$
\begin{aligned}
& p_{\max }\left(A_{j}\right)=p_{\min }\left(A_{j}\right)+\sum_{i=1}^{7} p\left(x_{i}\right) \eta_{A_{j}}\left(x_{i}\right) \text { as: } \\
& \quad p_{\max }\left(A_{1}\right)=0.8506 ; p_{\max }\left(A_{2}\right)=0.804 ; p_{\max }\left(A_{3}\right)=0.8428 ; p_{\max }\left(A_{4}\right)=0.9406 ;
\end{aligned}
$$

and the maximum probability of non-occurrence as follows:

$$
q_{\max }\left(A_{1}\right)=0.14 ; q_{\max }\left(A_{2}\right)=0.1687 ; q_{\max }\left(A_{3}\right)=0.3473 ; q_{\max }\left(A_{4}\right)=0.104 .
$$

- Step 6 Further, we find the value of probability of occurrence $p\left(A_{j}\right)=\frac{p_{\max }\left(A_{j}\right)+p_{\min }\left(A_{j}\right)}{2}$ as follows:

$$
p\left(A_{1}\right)=0.6246 ; p\left(A_{2}\right)=0.8213 ; p\left(A_{3}\right)=0.7239 ; p\left(A_{4}\right)=0.8288 ;
$$

and probability of non-occurrence $q\left(A_{j}\right)=\frac{q_{\max }\left(A_{j}\right)+q_{\min }\left(A_{j}\right)}{2}$ as follows:

$$
q\left(A_{1}\right)=0.1243 ; q\left(A_{2}\right)=0.159 ; q\left(A_{3}\right)=0.211 ; q\left(A_{4}\right)=0.0827 .
$$

- Step 7 Finally, by using the equation (3.6), we compute the determining probabilistic picture distance measures of all the known classes of the building materials $A_{1}, A_{2}, A_{3}$ and $A_{4}$ from the unknown class of building material $B$ as:

$$
d_{p}\left(A_{1}, B\right)=0.7652, d_{p}\left(A_{2}, B\right)=0.6496, d_{p}\left(A_{3}, B\right)=0.6419, d_{p}\left(A_{4}, B\right)=0.1728 .
$$

On the basis of the above obtained values of the distances, we conclude that the unknown material $B$ belongs to the known class $A_{4}$.

\subsection{Mineral classification problem}

In an another similar case of classification problem where we have 4 different minerals fields which are represented by the picture fuzzy sets $A_{1}, A_{2}, A_{3}, A_{4}$ and each of these fields is featured by the content of 6 minerals in the form of feature sample space $X=$ $\left\{x_{1}, x_{2}, x_{3}, x_{4}, x_{5}, x_{6}\right\}$. We consider an unknown mineral field $B$ which is to be classified among the known 4 classes. The data for this problem have also been from the similar application problem (refer Table 3 in [36]). In this type of problems, the aim is to identify the class of unknown mineral using the degree of distance measure of unknown material with that of known mineral along with the incorporation of probability of occurence/nonoccurrence of fuzzy event.

Applying the procedural steps (Step 1 to Step 7) of the proposed algorithm over the above stated problem, we compute and tabulate the probabilities of occurrence $p_{i}(i=$ $1,2, \ldots, 6)$ and probabilities of non-occurrence $q_{i}(i=1,2, \ldots, 6)$ as follows: 


\begin{tabular}{|c|c|c|c|c|c|c|c|}
\hline$x_{i}$ & $i=1$ & $i=2$ & $i=3$ & $i=4$ & $i=5$ & $i=6$ & Sum \\
\hline Probability of occurrence $\left(p\left(x_{i}\right)\right)$ & 0.51961 & 0.06863 & 0.01961 & 0.07843 & 0.14706 & 0.16667 & 1 \\
\hline Probability of non-occurrence $\left(q\left(x_{i}\right)\right)$ & 0.0 & 0.2 & 0.3333 & 0.1333 & 0.3333 & 0.0 & 1 \\
\hline
\end{tabular}

Next, by using the equation (3.6), we compute the determining probabilistic picture distance measures of all the known classes of the mineral fields $A_{1}, A_{2}, A_{3}$ and $A_{4}$ from the unknown class of mineral field $B$ as:

$$
d_{p}\left(A_{1}, B\right)=0.61435, d_{p}\left(A_{2}, B\right)=0.64141, d_{p}\left(A_{3}, B\right)=0.63187, d_{p}\left(A_{4}, B\right)=0.09446 .
$$

On the basis of the above obtained values of the distances, we conclude that the unknown mineral field $B$ belongs to the known class $A_{4}$.

\subsection{Financial investment risk analysis}

In this application problem, the main objective is to determine the best financial investment alternative from various available alternatives under the enterprise financial risk environment. In order to choose the most desirable alternative, the desirability levels of the five possible financial investment alternatives $A_{j}(j=1,2,3,4,5)$ are being evaluated. The team of decision makers/experts must make a decision according to the following four criteria: $G_{1}$ - the market risk; $G_{2}$ - the enterprise's operation and management risk; $G_{3}$ - the enterprise's assets structure risk; and $G_{4}$ - the environmental risk. The experts, on the basis of the above criteria, evaluate the five possible financial investment alternatives $A_{j}(j=1,2,3,4,5)$ by constructing the decision matrix using the picture fuzzy numbers represented in the following Table 1 .

Table 1. Decision matrix

\begin{tabular}{|c|c|c|c|c|}
\hline & $G_{1}$ & $G_{2}$ & $G_{3}$ & $G_{4}$ \\
\hline$A_{1}$ & $\begin{array}{llll}0.46 & 0.36 & 0.1)\end{array}$ & $\left(\begin{array}{lll}0.79 & 0.02 & 0.01)\end{array}\right.$ & $\left(\begin{array}{lll}0.43 & 0.45 & 0.08\end{array}\right)$ & $\left.\begin{array}{llll} & 0.18 & 0.39 & 0.04\end{array}\right)$ \\
\hline$A_{2}$ & $\left(\begin{array}{lll}0.43 & 0.32 & 0.18\end{array}\right)$ & $\left(\begin{array}{lll}0.73 & 0.04 & 0.11\end{array}\right)$ & (0.03 0.620 .33$)$ & $\left(\begin{array}{lll}0.53 & 0.25 & 0.18\end{array}\right)$ \\
\hline$A_{3}$ & $\left(\begin{array}{lll}0.71 & 0.23 & 0.01\end{array}\right)$ & $\left(\begin{array}{lll}0.87 & 0.02 & 0.03\end{array}\right)$ & $\left(\begin{array}{lll}0.04 & 0.55 & 0.3\end{array}\right)$ & $\left(\begin{array}{lll}0.48 & 0.26 & 0.16\end{array}\right)$ \\
\hline$A_{4}$ & $\left(\begin{array}{lll}0.25 & 0.4 & 0.15\end{array}\right)$ & $\left(\begin{array}{lll}0.64 & 0.12 & 0.13\end{array}\right)$ & $\left(\begin{array}{lll}0.01 & 0.69 & 0.25\end{array}\right)$ & $\left(\begin{array}{lll}0.02 & 0.54 & 0.26\end{array}\right)$ \\
\hline$A_{5}$ & $\left(\begin{array}{lll}0.5 & 0.45 & 0.03\end{array}\right)$ & $\left(\begin{array}{lll}0.78 & 0.03 & 0.11\end{array}\right)$ & $\left(\begin{array}{lll}0.03 & 0.5 & 0.26\end{array}\right)$ & $\left(\begin{array}{lll}0.13 & 0.65 & 0.19\end{array}\right)$ \\
\hline
\end{tabular}

Now, applying the procedural steps of the proposed algorithm over the above stated problem, we obtain:

- Step 1 We first determine the minimum membership value and non-membership value from the set of membership values \& non-membership values assigned by the experts, $A_{j}(j=1,2, \ldots, 5)$ to the objects $G_{i}(i=1,2, \ldots, 4)$, that is,

$$
\min _{j} \mu_{A_{j}}\left(G_{i}\right)=\mu^{\prime}\left(G_{i}\right) \text { and } \min _{j} \nu_{A_{j}}\left(G_{i}\right)=\nu^{\prime}\left(G_{i}\right) .
$$

For example, here for $i=1$, we have $\min _{j=1}^{5} \mu_{A_{j}}\left(G_{1}\right)=0.25$ and $\min _{j=1}^{5} \nu_{A_{j}}\left(G_{1}\right)=0.01$.

- Step 2 Next, we collect all the minimum membership values and non-membership values for each $G_{i}$ for $i=1,2, \ldots, 4$ to form the set $E=\left\{\mu\left(G_{1}\right), \mu\left(G_{2}\right), \ldots, \mu\left(G_{4}\right)\right\}$ and $E^{\prime}=$ $\left\{\nu\left(G_{1}\right), \mu\left(G_{2}\right), \ldots, \mu\left(G_{4}\right)\right\}$ as follows:

$$
E=\{0.25,0.64,0.01,0.02\}
$$

and

$$
E^{\prime}=\{0.01,0.01,0.08,0.04\}
$$


- Step 3 In this step, we evaluate the sum $S=\sum_{i=1}^{4} \mu^{\prime}\left(G_{i}\right)=0.92$ and $S^{\prime}=\sum_{i=1}^{4} \nu^{\prime}\left(G_{i}\right)=$ 0.14 and determine the probability of occurrence $p\left(G_{i}\right)=\frac{\mu^{\prime}\left(G_{i}\right)}{S}$ and the probability of non-occurrence $q\left(G_{i}\right)=\frac{\nu^{\prime}\left(G_{i}\right)}{S^{\prime}}$ as follows:

$$
p\left(G_{1}\right)=0.271739 ; p\left(G_{2}\right)=0.695652 ; p\left(G_{3}\right)=0.01087 ; p\left(G_{4}\right)=0.21739 ;
$$

and

$$
q\left(G_{1}\right)=0.071429 ; q\left(G_{2}\right)=0.071429 ; q\left(G_{3}\right)=0.571429 ; q\left(G_{4}\right)=0.285714 .
$$

- Step 4 Then, we compute the minimum probability of occurrence as:

$$
\begin{aligned}
& p_{\text {min }}\left(A_{1}\right)=0.683152 ; p_{\min }\left(A_{2}\right)=0.636522 ; p_{\text {min }}\left(A_{3}\right)=0.809022 ; \\
& p_{\text {min }}\left(A_{4}\right)=0.513696 ; p_{\min }\left(A_{5}\right)=0.68163 ;
\end{aligned}
$$

and the minimum probability of non-occurrence as:

$$
\begin{aligned}
& q_{\min }\left(A_{1}\right)=0.065 ; q_{\min }\left(A_{2}\right)=0.260714 ; q_{\min }\left(A_{3}\right)=0.22 \\
& q_{\min }\left(A_{4}\right)=0.237143 ; q_{\min }\left(A_{5}\right)=0.212857
\end{aligned}
$$

- Step 5 In this step, we compute the maximum probability of occurrence as:

$$
\begin{aligned}
& p_{\max }\left(A_{1}\right)=0.808261 ; p_{\max }\left(A_{2}\right)=0.763478 ; p_{\max }\left(A_{3}\right)=0.897065 ; \\
& p_{\max }\left(A_{4}\right)=0.725109 ; p_{\max }\left(A_{5}\right)=0.844348 ;
\end{aligned}
$$

and the minimum probability of non-occurrence as:

$$
\begin{aligned}
& q_{\max }\left(A_{1}\right)=0.217857 ; q_{\max }\left(A_{2}\right)=0.297143 ; q_{\max }\left(A_{3}\right)=0.320714 ; \\
& q_{\max }\left(A_{4}\right)=0.339286 ; q_{\max }\left(A_{5}\right)=0.348571 .
\end{aligned}
$$

- Step 6 Next, we find the value of probability of occurrence as: $p\left(A_{1}\right)=0.745707 ; p\left(A_{2}\right)=0.70 ; p\left(A_{3}\right)=0.853043 ; p\left(A_{4}\right)=0.619402 ; p\left(A_{5}\right)=0.762989 ;$ and probability of non-occurrence as:

$q\left(A_{1}\right)=0.141429 ; q\left(A_{2}\right)=0.278929 ; q\left(A_{3}\right)=0.270357 ; q\left(A_{4}\right)=0.288214 ; q\left(A_{5}\right)=0.280714$.

- Step 7 In view of the equation 3.6, we compute the determining probabilistic picture distance measure between the alternatives $A_{j} j=1,2, \ldots 5$ and the ideal solution $A^{+}$ which given by $A^{+}=\{(1,0,0),(1,0,0),(1,0,0),(1,0,0)\}$ as:

$$
\begin{aligned}
& d_{p}\left(A_{1}, A^{+}\right)=0.7281, d_{p}\left(A_{2}, A^{+}\right)=0.7609, d_{p}\left(A_{3}, A^{+}\right)=0.7298, \\
& d_{p}\left(A_{4}, A^{+}\right)=0.9453, d_{p}\left(A_{5}, A^{+}\right)=0.8679 .
\end{aligned}
$$

Hence, the ranking order achieved is as follows:

$$
A_{1}>A_{3}>A_{2}>A_{5}>A_{4} .
$$

On the basis of the above obtained values of the distances, we conclude that the alternative $A_{1}$ is the best among all.

\section{Results and discussions}

In order to ensure the viability of the proposed methodology, we study the obtained results of the illustrative examples in this section. The proposed idea of composing the probabilistic behavior of randomness and four different parameters of picture fuzzy uncertainty to define the novel distance measure for picture fuzzy sets is certainly an advanced extension in the direction of research. This gives an added advantage to deal with a wider sense of applicability in uncertain situations. We present some important comparative remarks and advantages of utilizing the proposed methodology below: 


\subsection{Comparative analysis with existing approaches}

In view of the building material classification problem discussed in the subsection 4.1, the following Table 2 shows the comparison between the closeness index values obtained by the proposed method and Wei's method [36]: Next, in view of the mineral classification

Table 2. Comparison table with respect to Wei's method (Example 1) [36]

\begin{tabular}{|c|c|c|c|c|}
\hline Measures & $\left(A_{1}, B\right)$ & $\left(A_{2}, B\right)$ & $\left(A_{3}, B\right)$ & $\left(A_{4}, B\right)$ \\
\hline$W_{P F S}^{1}$ & 4 & 3 & 2 & 1 \\
\hline$W_{P F S}^{2}$ & 4 & 3 & 2 & 1 \\
\hline$W_{P F S}^{3}$ & 4 & 3 & 2 & 1 \\
\hline Proposed Method & 4 & 2 & 3 & 1 \\
\hline
\end{tabular}

problem discussed in the subsection 4.2, the following Table 3 shows the comparison between the closeness index values obtained by the proposed method and Wei's method [36]:

Table 3. Comparison table with respect to Wei's method (Example 2) [36]

\begin{tabular}{|c|c|c|c|c|}
\hline Measures & $\left(A_{1}, B\right)$ & $\left(A_{2}, B\right)$ & $\left(A_{3}, B\right)$ & $\left(A_{4}, B\right)$ \\
\hline$W_{P F S}^{1}$ & 2 & 4 & 3 & 1 \\
\hline$W_{P F S}^{2}$ & 2 & 4 & 3 & 1 \\
\hline$W_{P F S}^{3}$ & 4 & 3 & 2 & 1 \\
\hline Proposed Method & 2 & 4 & 3 & 1 \\
\hline
\end{tabular}

Thus, in contrast with the Wei's method [36], the comparison tables (Table 2 and Table 3 ) and the analysis clearly highlight the competency and applicability of the proposed methodology with an acceptability to the probabilistic framework in the picture fuzzy distance measure. Therefore, the additional feature of the proposed probabilistic distance measure is also capable, reliable, more authentic and consistent enough.

Further, in view of the financial investment risk problem discussed in the subsection 4.3, the following Table 4 shows the comparison between the ranking obtained by the proposed method, Wang et al. method [33] and Wei's method [35]:

Table 4. Comparison of ranking order with respect to different approaches

\begin{tabular}{|c|c|c|c|c|c|}
\hline Approaches & $A_{1}$ & $A_{2}$ & $A_{3}$ & $A_{4}$ & $A_{5}$ \\
\hline Wang et al. method [33] & 1 & 4 & 2 & 5 & 3 \\
\hline Wei's method [35] & 1 & 3 & 2 & 5 & 4 \\
\hline Proposed Method & 1 & 3 & 2 & 5 & 4 \\
\hline
\end{tabular}

On the basis of the values obtained for the measures, it may be observed that the ranking order as per Wang et al. method [33] and Wei's method [35] is equally consistent with the results of the proposed measure. Therefore, the additional feature of the proposed probabilistic distance measure is also capable, reliable, more authentic and consistent enough.

\section{Remarks:}

- The applicability of the proposed methodology appears to be more prominent in the probabilistic framework and the analysis carried above clearly depicts the consistency and authenticity.

- The additional feature of the proposed probabilistic distance measure is well consistent and has the advantage of spanning the uncertain information in a more precise way.

- The application areas which have been discussed are the recent developments and the proposed methodology can further be implemented in other areas of contemporary research and evaluation. 


\subsection{Advantages of the proposed measure}

- In case of practical circumstances, the uncertainty space can be categorized into two subspaces, i.e., vagueness and non-vagueness space (Refer Figure 3). The information that is not present precisely is represented in the vagueness subspace and the information which is acknowledged by the people/experts is contained in non-vagueness subspace. It may be noted that the uncertainty in the non-vagueness subspace is an undetermined property. By considering the refusal and hesitancy there are two opposite viewpoints about the belongingness of the element to the set. Thus, it is appropriate to explore and deal the uncertainty from vagueness and non-vagueness.

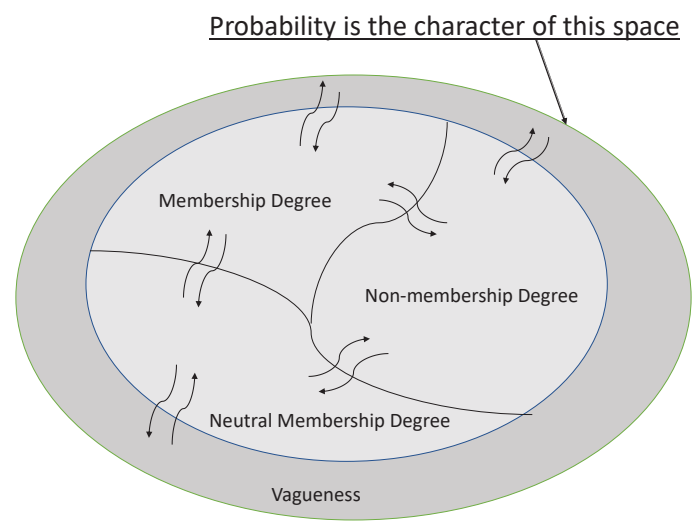

Figure 3. Uncertainty space: Vagueness subspace and non-vagueness subspace

- It may be noted that the picture fuzzy set can handle the uncertainty in a better way than the intuitionistic fuzzy set/Pythagorean fuzzy set. The proposed composite probabilistic distance measure can be considered as the generalized distance measure because it incorporates all the four components of fuzziness (i.e., membership, non-membership, neutral membership and refusal).

- The drawback in the existing literature of the intuitionistic fuzzy sets and Pythagorean fuzzy sets is that their conditions do not allow the experts/decision makers to allocate the membership values of their own choice (Refer Table 5). Somehow, this makes the decision makers bounded for providing their input in a particular domain. However, the proposed distance measure for picture fuzzy sets provide a generalization feature which may give a strong impact in an application/decision making process.

Table 5. Need to address the problem arises in IFSs and PyFSs

\begin{tabular}{|l|c|c|c|c|}
\hline & $C_{1}$ & $C_{2}$ & $C_{3}$ & $C_{4}$ \\
\hline$C_{1}$ & $(1.0+0.0+0.0=1)$ & $(0.40+0.20+0.40=1)$ & $(0.30+0.15+0.45<1)$ & $(0.56+0.10+0.27<1)$ \\
$C_{2}$ & $(0.30+0.20+0.44<1)$ & $(1.0+0.0+0.0=1)$ & $(0.40+0.14+0.46<1)$ & $(0.51+0.29+0.61<1)$ \\
$C_{3}$ & $(0.56+0.20+0.16<1)$ & $(0.44+0.24+0.22<1)$ & $(1.0+0.0+0.0=1)$ & $(0.48+0.14+0.22<1)$ \\
$C_{4}$ & $(0.49+0.17+0.24<1)$ & $(0.59+0.09+0.20<1)$ & $(0.55+0.17+0.10<1)$ & $(1.0+0.0+0.0=1)$ \\
\hline
\end{tabular}

\section{Conclusions and scope for future work}

The idea of composite probabilistic picture fuzzy distance measure has been successfully introduced with a justified framework for incorporating the probabilistic randomness and the four fuzziness parameters (membership, neutral membership, non-membership and refusal). The proposed algorithm based on the picture distance measure has been 
well implemented for solving the classification problems - building material and mineral classification problem under consideration and a decision making problem of financial investment risk. The comparative analysis under the each application problem and the advantages discussed in the results section clearly indicate that the feature of the proposed probabilistic distance measure is additionally helpful, reliable, more authentic and consistent enough.

The proposed notion and the algorithm using the aggregation operators may further be utilized and extended in future with the following possibilities:

- The employee selection process used by human resource management in an organization may be categorized into 4 types of category - true positive, true negative, false negative and false positive which may be understood to be equivalent to positive, neutral, negative and refusal degrees of picture fuzzy set.

- The proposed measures can be applied to various other classification problems where the uncertainty is prominent such as medical diagnosis, terrain classification, statistical data analysis, clustering, weather forecasting, etc.

- The proposed measure advances a small inclination toward the development of similarity measures in advanced fuzzy sets i.e., picture fuzzy sets.

- The concept of 'useful' picture fuzzy information in reference with utility distribution can further be integrated in terms of cross entropy/divergence to deal with the picture fuzzy uncertainty and probabilistic useful randomness.

- Mahmood et al. [25] introduced the notion of spherical fuzzy set which gives an additional strength to the idea of the picture fuzzy set by broadening/enlarging the space for the grades of all the four parameters. Utilizing this notion, the proposed probabilistic distance measure can further be extended to handle the limitation of the picture fuzzy set which arises in terms of the constraint related to the uncertainty parameters.

Acknowledgment. We are very much thankful to the anonymous reviewers for suggesting the necessary points/mistakes which have been duly implemented/ corrected for the necessary improvement of the manuscript. We sincerely acknowledge our deep sense of gratitude to the reviewers and Editor-in-Chief for giving their valuable time to the manuscript. We are very much grateful to our parent university (JUIT, Waknaghat) for providing us the platform and environment to carry out this research.

\section{References}

[1] K.T. Atanassov, Intuitionistic fuzzy sets, Fuzzy Sets and Systems 20, 87-96, 1986.

[2] K.T. Atanassov, Geometrical interpretation of the elements of the intuitionistic fuzzy objects, Int. J. Bioautomation 20 (1), S27-S42, 2016.

[3] R.K. Bajaj and A. Guleria, Dimensionality reduction technique in decision making using Pythagorean fuzzy soft matrices, Recent Advances in Computer Science and Communications (Formerly: Recent Patents on Computer Science) 13 (3), 406-413, 2020.

[4] P. Burillo and H. Bustince, Entropy on intuitionistic fuzzy sets and on interval-valued fuzzy sets, Fuzzy Sets and Systems 78, 305-316, 1996.

[5] B.C. Cuong, Picture fuzzy sets-first results. Part 1, in preprint of seminar on NeuroFuzzy Systems with Applications, Institute of Mathematics, 2013.

[6] B.C. Cuong, Picture fuzzy sets, Journal of Computer Science and Cybernetics 30, 409-420, 2014.

[7] N.V. Dinh, N.X. Thao, and N.M. Chau, Distance and dissimilarity measure of picture fuzzy sets, in Conf. FAIR 10, 104-109, 2017.

[8] P. Dutta, Medical diagnosis via distance measures on picture fuzzy sets, Advances in Modelling and Analysis 54, 137-152, 2017. 
[9] F. Feng, H. Fujita, M. Irfan Ali, R.R. Yager, and X. Liu, Another view on generalized intuitionistic fuzzy soft sets and related multiattribute decision making methods, IEEE Trans Fuzzy Syst 27 (3), 474-488, 2019.

[10] F. Feng, M. Liang, H. Fujita, R.R. Yager, and X. Liu, Lexicographic orders of intuitionistic fuzzy values and their relationships, Mathematics 7 (2), 166, 2019.

[11] F. Feng, Z. Xu, H. Fujita, and M. Liang, Enhancing PROMETHEE method with intuitionistic fuzzy soft sets, Int. J. Intell. Syst 35, 1071-1104, 2020.

[12] F. Feng, Y. Zheng, J.C.R. Alcantud, and Q. Wang, Minkowski weighted score functions of intuitionistic fuzzy values, Mathematics 8, 1143, 2020.

[13] H. Garg and G. Kaur, Novel distance measures for cubic intuitionistic fuzzy sets and their applications to pattern recognitions and medical diagnosis, Granular Computing 5, 169-184, 2020.

[14] A. Guleria and R.K. Bajaj, On Pythagorean fuzzy soft matrices, operations and their applications in decision making and medical diagnosis, Soft Comput. 23, 7889-7900, 2019 .

[15] A. Guleria and R.K. Bajaj, Pythagorean fuzzy $(R, S)$-norm discriminant measure in various decision making processes, J. Intell. Fuzzy Syst. 38, 761-777, 2020.

[16] A. Guleria and R.K. Bajaj, A robust decision Making Approach for hydrogen power plant site selection utilizing $(R, S)$-norm Pythagorean fuzzy information measures based on VIKOR and TOPSIS method, Int. J. Hydrog. Energy 45 (38), 18802-18816, 2020.

[17] A.G. Hatzimichailidis, G.A. Papakostas, and V.G. Kaburlasos, A novel distance measure of intuitionistic fuzzy sets and its application to pattern recognition problems, Int. J. Intell. Syst. 27, 396-409, 2012.

[18] W.L. Hung and M.S. Yang, Similarity measures of intuitionistic fuzzy sets based on hausdorff distance, Pattern Recognit. Lett. 25, 1603-1611, 2004.

[19] W.L. Hung and M.S. Yang, Similarity measures of intuitionistic fuzzy sets based on LP metric, Internat. J. Approx. Reason. 46, 120-136, 2006.

[20] C. Jana and M. Pal, Assessment of enterprise performance based on picture fuzzy hamacher aggregation operators, Symmetry 11, 75, 2019.

[21] C. Jana, T. Senapati, M. Pal, and R.R. Yager, Picture fuzzy Dombi aggregation operators: Application to MADM process, Appl. Soft Comput. 74, 99-109, 2019.

[22] A.M. Khalil, S.G. Li, H. Garg, H. Li, and S. Ma, New operations on interval-valued picture fuzzy set, interval-valued picture fuzzy soft set and their applications, IEEE Access 7, 51236-51253, 2019.

[23] D. Li and W. Zeng, Distance measure of Pythagorean fuzzy sets, Int. J. Intell. Syst. 33, 348-361, 2018.

[24] M. Luo and R. Zhao, A distance measure between intuitionistic fuzzy sets and its application in medical diagnosis, Artif Intell Med 89, 34-39, 2018.

[25] T. Mahmood, U. Kifayat, Q. Khan, and N. Jan, An approach toward decision making and medical diagnosis problems using the concept of spherical fuzzy sets, Neural. Comput. Appl. 31, 7041-7053, 2019.

[26] G.A. Papakostas, A.G. Hatzimichailidis, and V.G. Kaburlasos, Distance and or similarity measures between intuitionistic fuzzy sets: A comparative analysis from a pattern recognition point of view, Pattern Recognit. Lett. 34, 1609-1622, 2013.

[27] L.H. Son, Generalized picture distance measure and applications to picture fuzzy clustering, Appl. Soft Comput. 46, 284-295, 2016.

[28] L.H. Son, Measuring analogousness in picture fuzzy sets: From picture distance measures to picture association measures, Fuzzy Optim. Decis. Mak. 16, 1-20, 2017.

[29] E. Szmidt and J. Kacprzyk, A concept of a probability of an intuitionistic fuzzy event, 1999 IEEE International Fuzzy Systems, Conference Proceedings, Seoul, South Korea 3, 1346-1349, 1999. 
[30] E. Szmidt and J. Kacprzyk, Distances between intuitionistic fuzzy sets, Fuzzy Sets and System 114, 505-518, 2000.

[31] P.H. Thong and L.H. Son, Picture fuzzy clustering for complex data, Eng. Appl. Artif. Intell. 56, 121-130, 2016.

[32] P.H. Thong and L.H. Son, A novel automatic picture fuzzy clustering method based on particle swarm optimization and picture composite cardinality, Knowl Based Syst. 109, 48-60, 2016.

[33] R. Wang, J. Wang, H. Gao, and G. Wei, Methods for MADM with picture fuzzy muirhead mean operators and their application for evaluating the financial investment risk, Symmetry 11, 6, 2019.

[34] G.W. Wei, Picture fuzzy cross-entropy for multiple attribute decision making problems, J. Bus. Econ. Manag. 17, 491-502, 2016.

[35] G.W. Wei, Picture fuzzy aggregation operators and their application to multiple attribute decision making, J. Intell. Fuzzy Syst. 33, 713-724, 2017.

[36] G.W. Wei, Some similarity measures for picture fuzzy sets and their applications,Iran. J. Fuzzy Syst. 15, 77-89, 2018.

[37] G.W. Wei and H. Gao, The generalized Dice similarity measures for picture fuzzy sets and their applications, Informatica (Vilnius) 29, 1-18, 2018.

[38] R.R. Yager, Pythagorean fuzzy subsets, in: Proceedings of Joint IFSA World Congress and NAFIPS Annual Meeting, Edmonton, Canada, 57-61, 2013.

[39] R.R. Yager, Pythagorean membership grades in multicriteria decision making, IEEE Trans Fuzzy Syst 22 (4), 958-965, 2014.

[40] R.R. Yager, Generalized orthopair fuzzy sets, IEEE Trans Fuzzy Syst 25 (5), 1222 1230, 2017.

[41] L.A. Zadeh, Fuzzy sets, Inf. Control. 8, 338-353, 1965.

[42] L.A. Zadeh, Probability measure of fuzzy events, J. Math. Anal. Appl. 23, 421-427, 1968.

[43] S. Zeng, S. Asharf, M. Arif, and S. Abdullah, Application of exponential jensen picture fuzzy divergence measure in multi-criteria group decision making, Mathematics 7, 191, 2019.

[44] X.L. Zhang and Z.S. Xu, Extension of TOPSIS to multi-criteria decisionmaking with Pythagorean fuzzy sets, Int. J. Intell. Syst. 29, 1061-1078, 2014. 